\title{
Colorectal Cancer Manifesting with Metastasis to Prolactinoma: Report of a Case Involving Symptoms Mimicking Pituitary Apoplexy
}

\author{
Yotsapon Thewjitcharoen ${ }^{1}$, Shanop Shuangshoti ${ }^{2}$, Sukalaya Lerdlum ${ }^{3}$, \\ Rungsak Siwanuwatn ${ }^{4}$ and Sarat Sunthornyothin ${ }^{1}$
}

\begin{abstract}
Pituitary metastasis is an uncommon first presentation of systemic malignancy. The most common presenting symptom of pituitary metastasis is diabetes insipidus reflecting involvement of the stalk and/or posterior pituitary. We herein present a unique case of the coexistence of both a functioning pituitary adenoma (prolactinoma) and pituitary metastasis of advanced colorectal cancer with pituitary apoplexy as the first manifestation of underlying malignancy. The present case emphasizes the need to consider pituitary metastasis as a differential diagnosis in patients presenting with pituitary lesions and be aware that tumor-to-tumor metastasis can occur unexpectedly in those with pituitary metastases.
\end{abstract}

Key words: pituitary metastasis, prolactinoma, apoplexy, colon cancer, tumor-to-tumor metastasis

(Intern Med 53: 1965-1969, 2014)

(DOI: 10.2169/internalmedicine.53.2353)

\section{Introduction}

Pituitary metastasis is a rare and uncommon presentation of systemic malignancy. The clinical and radiologic features of lesions of pituitary metastasis and adenoma are indistinguishable. Although pituitary apoplexy usually occurs in patients with pre-existing pituitary macroadenoma, it has also been described in those with a normal pituitary gland, craniopharyngioma, lymphocytic hypophysitis and pituitary metastasis (1). In rare circumstances, these two conditions may be detected simultaneously (2-4). Regarding metastasis to pituitary adenoma, this condition is an extremely rare phenomenon in which one tumor type serves as the recipient for a second, biologically unrelated, primary donor tumor within the same patient.

We herein present a unique case of the coexistence of a functioning pituitary adenoma (prolactinoma) and pituitary metastasis of advanced colorectal cancer. The patient was a 65-year-old man who first presented with signs and symptoms simulating pituitary apoplexy. The case emphasizes the need to consider pituitary metastasis in the differential diagnosis of pituitary lesions, as this condition may be the first presentation of the underlying cancer.

\section{Hormone measurements and immunohistochemistry}

The serum cortisol, prolactin and thyroid hormone levels were measured using a two-site immunoradiometric assay (IRMA) (Siemens, Deerfield, USA.) An immunohistochemical study of prolactin, CDX-2, CK-7 and CK-20 was performed using standard techniques with formalin-fixed, paraffin-embedded sections of tumor specimens collected during surgery.

Informed consent was obtained from a relative of the patient for educational purposes.

\footnotetext{
${ }^{1}$ Division of Endocrine and Metabolism, Department of Internal Medicine, Faculty of Medicine, Chulalongkorn University, Thailand, ${ }^{2}$ Department of Pathology and Chulalongkorn GenePRO Center, Faculty of Medicine, Chulalongkorn University, Thailand, ${ }^{3}$ Department of Radiology, Faculty of Medicine, Chulalongkorn University, Thailand and ${ }^{4}$ Division of Neurosurgery, Department of Surgery, Faculty of Medicine, Chulalongkorn University, Thailand

Received for publication December 18, 2013; Accepted for publication March 3, 2014

Correspondence to Dr. Yotsapon Thewjitcharoen, kamijoa@hotmail.com
} 

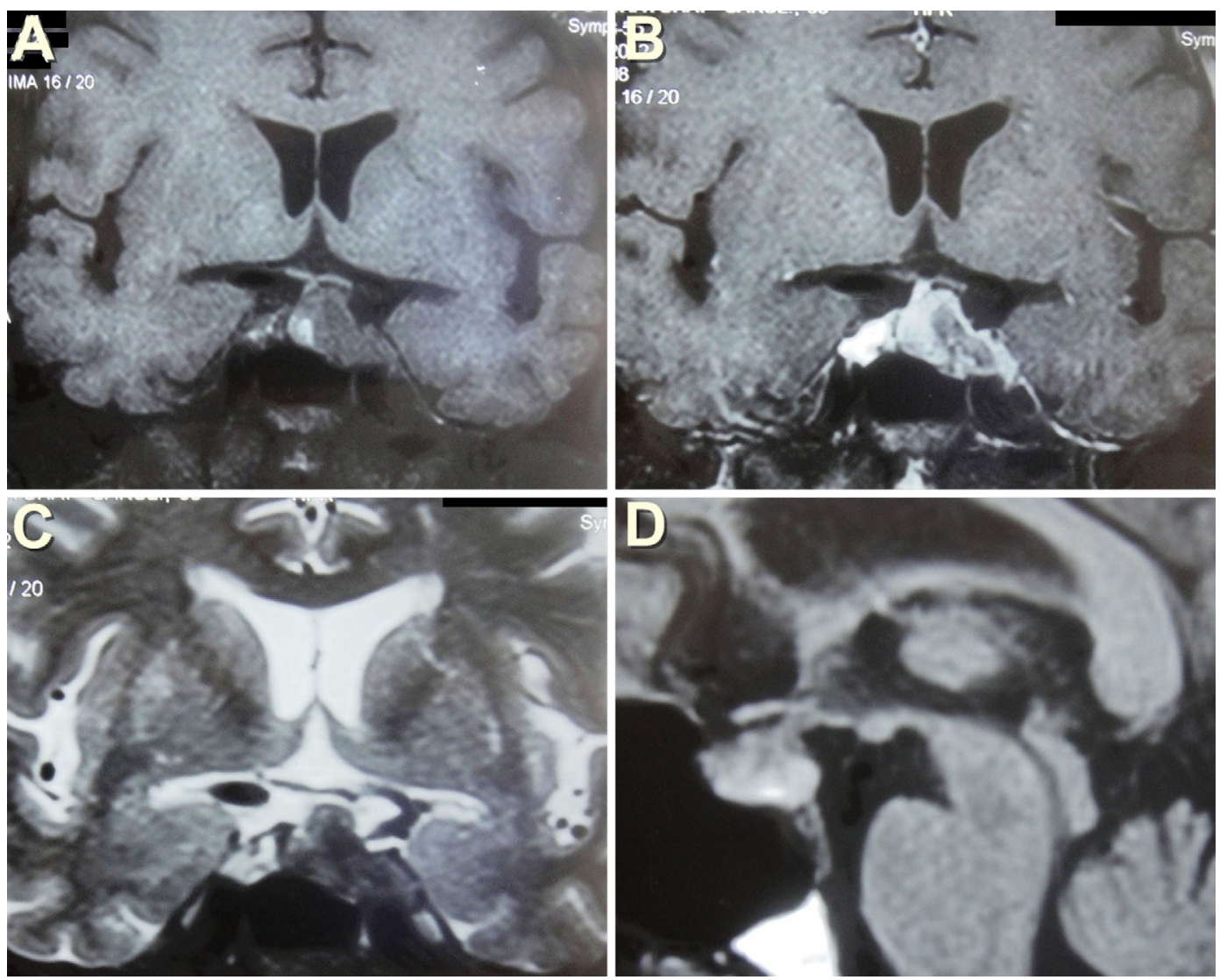

Figure 1. MRI study performed four weeks after the onset of headache. A: A T1-weighted coronal image shows an inhomogeneous isointense sellar mass that had invaded the left cavernous sinus; B: A gadolinium-enhanced T1-weighted coronal image demonstrates a less enhanced area in the sellar mass; C: A T2-weighted coronal image shows a hypointense mass; D: A T1-weighted sagittal image reveals a normal pituitary stalk and preserved posterior bright spot.

\section{Case Report}

A 65-year-old Thai man presented with the sudden onset of left eye ptosis and a visual disturbance. A severe headache, more so in the left retro-orbital area, began soon afterward, followed by nausea and lightheadedness upon standing. The patient denied fever, diarrhea or polyuria. He also reported a decrease in libido within the past few years. He went to see a general practitioner near his hometown for three weeks, without receiving a definitive diagnosis. The patient's headache continued to worsen, and his vision in the left eye deteriorated during the fourth week after the initial onset of symptoms. Without any improvements in symptoms, he was referred to us by an ophthalmologist who suspected an intracranial lesion based on the left eye ptosis and severe headache.

Magnetic resonance imaging (MRI) performed four weeks after onset demonstrated a $2.0 \times 2.3 \times 1.7-\mathrm{cm}$ sellar mass that had invaded the left cavernous sinus (Fig. 1). Pre-contrast T1-weighted images (T1WI) showed an inhomogeneous hypointensemass that additionally exhibited hypointensitysignals on T2-weighted images (T2WI). The lesion became inhomogeneously enhanced following gadolinium injection. In addition, a normal pituitary stalk with a preserved posterior bright spot was observed. The patient was therefore transferred to King Chulalongkorn Memorial Hospital with a presumptive diagnosis of pituitary apoplexy.

Upon examination, the patient was found to be drowsy, with a pulse rate of $74 / \mathrm{min}$ and a blood pressure of $95 / 60$ $\mathrm{mmHg}$. An impaired medial gaze and left eye blindness with complete ptosis were observed, indicating involvement of the left cranial nerves II and III. No gynecomastia or galactorrhea was observed. Blood samples were collected for hormonal studies, and a stress dose of intravenous hydrocortisone was administered immediately for presumed secondary adrenal insufficiency. Over the next 12 hours, the patient's condition improved under steroid treatment; however, the complete ptosis of the left eye persisted. A subsequent anterior pituitary function test showed a prolactin (with dilution of 1:100) level of $254 \mathrm{ng} / \mathrm{mL}$ (normal range, $2-25 \mathrm{ng} / \mathrm{mL}$ ), free T4 level of $0.6 \mathrm{ng} / \mathrm{dL}$ (normal range, $0.8-1.8 \mathrm{ng} / \mathrm{dL}$ ), random cortisol level at $1.00 \mathrm{PM}$ of $22 \mu \mathrm{g} / \mathrm{dL}$, folliclestimulating hormone level of $0.4 \mathrm{IU} / \mathrm{L}$ (normal range, 1.0$8.4 \mathrm{IU} / \mathrm{L}$ ), luteinizing hormone level of $0.7 \mathrm{IU} / \mathrm{L}$ (normal range, 1.0-10.5 IU/L), total testosterone level of $0.2 \mathrm{nmol} / \mathrm{L}$ (normal range, 5.9-24.7 nmol/L) and IGF-1 level of less than $25 \mathrm{ng} / \mathrm{mL}$. Based on his clinical presentation and hor- 


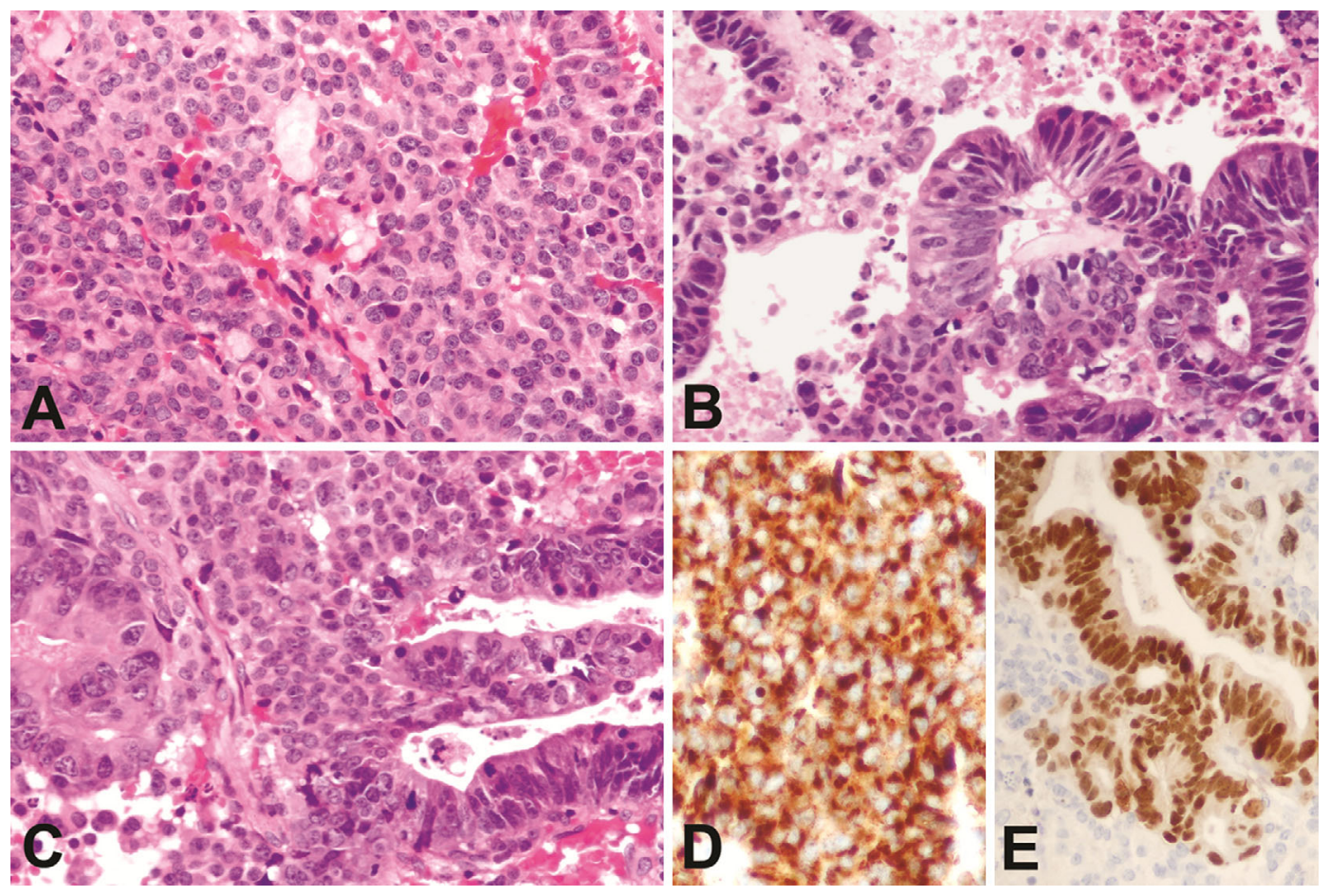

Figure 2. Pathology of the metastatic colonic adenocarcinoma in prolactinoma. Monotonous round adenoma cells with a stippled chromatin pattern (A) and colonic adenocarcinoma with dirty necrosis (B) are shown. In several areas, the pituitary adenoma and adenocarcinoma are intermixed (C). According to the immunohistochemical study, the endocrine tumor cells express prolactin (D), while the malignant glandular cells are stained positively for CDX-2 (E). A-C, Hematoxylin and Eosin staining; D, Prolactin immunostaining, and E, CDX-2 immunostaining.

mone profile, the patient was initially diagnosed as having pituitary apoplexy with panhypopituitarism due to prolactinoma. The prolactin level, however, was not as high as expected in patients with usual prolactin-producing macroadenomas. Therefore, non-functioning pituitary macroadenoma was included in the differential diagnosis.

The patient underwent subtotal tumor removal via transsphenoidal surgery. A lobulated and sticky intrasellar tumor was found adhering to the left internal carotid artery. No hemorrhage was observed. Postoperatively, the prolactin level declined to $59 \mathrm{ng} / \mathrm{mL}$ at two weeks and to normal (24 $\mathrm{ng} / \mathrm{mL}$ ) at one month. The morning serum cortisol level was reevaluated three days after the operation, showing an adequate response (8 AM serum cortisol level, $21 \mu \mathrm{g} / \mathrm{dL}$ ). Therefore, the dose of intravenous steroids was gradually tapered. The patient's vision returned to normal, although the ptosis of the left eye persisted. No diabetes insipidus was noted.

A pathological examination showed two distinct but intermixed populations of tumor cells involving pituitary adenoma and metastatic adenocarcinoma (Fig. 2). The adenoma cells exhibited monotonous round nuclei, a stippled chromatin pattern and eosinophilic cytoplasm. With respect to the carcinomatous component, hyperchromatic and pleomorphic columnar cells were detected, forming distinctive glands that were admixed with dirty necrosis. The adenoma cells expressed chromogranin and prolactin; the remaining adenohy- pophyseal hormones were non-reactive. Meanwhile, the malignant glandular cells were reactive for CDX-2 and CK20 but negative for $\mathrm{CK} 7$, thus suggesting a colorectal origin.

On direct questioning, the patient admitted to having intermittent bleeding per the rectum during the preceding years andhad experienced rapid weight loss of $30 \mathrm{~kg}$ over the past two months, although he denied a history of abdominal pain or changes in bowel habits. A further work up revealed a $10-\mathrm{cm}$ mass in the sigmoid colon with multiple liver metastases and several tinylung nodules. A biopsy of the mass in the sigmoid colon confirmed the diagnosis of well-differentiated adenocarcinoma. The patient received palliative chemotherapy and ultimately succumbed to the disease nine months after diagnosis.

\section{Discussion}

Metastasis to the pituitary gland is uncommon, especially in cases of symptomatic pituitary metastasis as the first presentation of systemic malignancy (1). Among symptomatic patients, diabetes insipidus is the most frequently reported finding, with a variable incidence ranging from $29 \%$ to $81 \%$ (2-4). Other presenting symptoms of pituitary metastasis include a mass effect or deficiency in the levels of anterior pituitary hormones.

Pituitary apoplexy is a clinical syndrome characterized by the sudden onset of headaches, vomiting, visual impairment 
and decreased consciousness. Although pituitary apoplexy usually occurs in patients with pre-existing pituitary macroadenoma, it has also been described in those with a normal pituitary gland, craniopharyngioma, lymphocytic hypophysitis and, in rare instances $(<5 \%)$, pituitary metastasis $(2,5,6)$. The major differential diagnoses of pituitary apoplexy include ruptured posterior communicating aneurysms, cavernous sinus thrombosis and hemorrhagic Rathke cleft cysts (6). Although the gradual worsening of symptoms observed in our patient is rather unusual for pituitary apoplexy, some cases of apoplexy with an atypical course (e.g., isolated third nerve palsy without headaches) have been documented (7).

A recent set of guidelines from the United Kingdom state that patients with apoplexy and significant neuro-ophthalmic signs or a reduced level of consciousness should undergo surgical decompression. However, it is unclear as to which criteria should be used to define a significant neuroophthalmic deficit (8), and the decision regarding the timing of surgery is remains a subject of debate in the management of pituitary apoplexy. In the present case, moderate hyperprolactinemia (prolactin level, $254 \mathrm{ng} / \mathrm{mL}$ ) may be a signal of the presumptive diagnosis of prolactinoma in which conservative management is recommended in cases involving stable pituitary apoplexy (8). However, the degree of hyperprolactinemia was not as high as expected when the large size of pituitary mass was taken into consideration. A nonfunctioning macroadenoma or other type of sellar mass may have causedthe patient's modest hyperprolactinemia by interrupting inhibitory hypothalamic dopaminergic transport. Therefore, the presumptive diagnosis of prolactinoma is doubtful. Surgical removal of the lesion was selected in this case in order to reduce the mass effect and identify the definitive cause of the patient's hyperprolactinemia. In retrospect, the pituitary metastasis may have been mistaken for a prolactinoma had a conservative approach been applied.

In a series of 52 patients with pituitary metastasis diagnosed at the Mayo clinic from 1950 to 1996, colon cancer was found to be the primary source of the disease in only three cases, and all cancers were discovered before the pituitary lesion (9). In another review, colorectal cancer was found to account only for $2.36 \%$ of lesions (9/380 pituitary metastases) (4). Regarding metastasisto pituitary adenoma, breast and lung malignancies are the most common primary neoplasms (3). In 13/18 cases of pituitary metastases to pituitary adenoma reported in the recent literature (10), the recipient tumor was a non-functioning adenoma; the remaining five cases included three patients with prolactinomas, and one patient each with ACTH-secreting and growth hormone-secreting adenomas. Metastasis of gastrointestinal origin (gastric cancer) to a prolactinoma, as observed in the present case, was definitively identified in only one previous case, reported by van Seters et al. in 1985 (11). To the best of our knowledge, the present case is the first case of colorectal cancer with metastasis to a pre-existing prolactinoma presenting with symptoms mimicking pituitary apoplexy as the manifestation of the underlying cancer. MRI findings that may favor pituitary metastasis include thickening of the pituitary stalk, loss of the posterior pituitary bright spot and isointense signals on both T1- and T2-weighted images, as suggested in one study (12). However, none of these findings were observed in our case.

The association between pituitary adenoma and pituitary metastasis is debatable. Patients with pituitary adenomas have been reported to have an increased risk of second malignancies (13). Metastatic seeding to pituitary adenoma may be related to an abnormal pituitary vasculature in areas adjacent to the adenoma or hormonal effects with the potential to enhance tumor cell proliferation (14). The slow metabolic rate of prolactinomasmay also provide a non-competitive environment for the growth of metastatic tumors (15). On the other hand, due to the rarity of tumor-to-tumor metastasis, this feature is believed to be simply a coincidental finding (16).

The prognosis of metastatic cancer to pituitary adenoma is grim, as most patients already have widespread metastases at the time of diagnosis (2). The mean survival after the development of pituitary metastasis is only six months, with an overall 1-year mortality rate of more than $90 \%$ (4). Therapeutic options are limited and include palliative radiotherapy, hormone replacement therapy when indicated and/or chemotherapy for the primary cancer.

In conclusion, we herein described the case of a unique patient who presented with a clinical diagnosis of pituitary apoplexy and elevated prolactin level. Prolactinoma was the initial presumptive diagnosis; however, subsequent pathological examinations documented the coexistence of a prolactinoma and metastatic colorectal adenocarcinoma. This case highlights the fact that pituitary metastasis can be the first manifestation of underlying malignancy and thus should always be included in the differential diagnosis of pituitary masses. Furthermore, this case emphasizes the importance of detailed and thorough history-taking and physical examinations.

\section{The authors state that they have no Conflict of Interest (COI).}

\section{References}

1. Teears RJ, Silverman EM. Clinicopathologic review of 88 cases of carcinoma metastatic to the pituitary gland. Cancer 36: 216-220, 1975.

2. Morita A, Meyer FB, Laws ER Jr. Symptomatic pituitary metastases. J Neurosurg 89: 69-73, 1998.

3. Fassett DR, Couldwell WT. Metastases to the pituitary gland. Neurosurg Focus 16: E8, 2004.

4. Komninos J, Vlassopoulou V, Protopapa D. Tumors metastatic to the pituitary gland: case report and literature review. J Clin Endocrinol Metab 89: 574-580, 2004.

5. Anderson DF, Afshar F, Toma N. Metastatic prostatic adenocarcinoma presenting as complete ophthalmoplegia from pituitary apoplexy. Br J Ophthalmol 78: 315-316, 1994.

6. Chhiber SS, Bhat AR, Khan SH. Apoplexy in sellar metastasis: a case report and review of literature. Turk Neurosurg 21: 230-234, 
2011.

7. Enatsu R, Asahi M, Matsumoto M. Pituitary apoplexy presenting atypical time course of ophthalmic symptoms. Tohoku J Exp Med 227: 59-61, 2012.

8. Schubiger O, Haller D. Metastases to the pituitary: hypothalamic axis. An MR study of 7 symptomatic patients. Neuroradiology 34: 131-134, 1992.

9. Rajasekaran S, Vanderpump M, Baldeweg S. UK guidelines for the management of pituitary apoplexy. Clin Endocrinol (Oxf) 74: 9-20, 2011.

10. Noga C, Prayson R, Kowalski R. Metastatic adenocarcinoma to a pituitary adenoma. Ann Diagn Pathol 5: 354-360, 2001.

11. Ramsay JA, Kovacs K, Scheithauer BW. Metastatic carcinoma to pituitary adenomas: a report of two cases. Exp Clin Endocrinol 92: 69-76, 1988.
12. van Seters AP, Bots GT, van Dulken H. Metastasis of an occult gastric carcinoma suggesting growth of a prolactinoma during bromocriptine therapy: a case report with a review of the literature. Neurosurgery 16: 813-817, 1985.

13. Hurley TR, D'Angelo CM, Clasen RA. Adenocarcinoma metastatic to a growth-hormone-secreting pituitary adenoma: case report. Surg Neurol 37: 361-365, 1992.

14. Molinatti PA, Scheithauer BW, Randall RV. Metastasis to pituitary adenoma. Arch Pathol Lab Med 109: 287-289, 1985.

15. Post KD, McCormick PC, Hays AP. Metastatic carcinoma to pituitary adenoma. Report of two cases. Surg Neurol 30: 286-292, 1988.

16. Heshmati H, Scheithauer S, Young WF. Metastases to the pituitary gland. Endocrinologist 12: 45-49, 2002.

(C) 2014 The Japanese Society of Internal Medicine http://www.naika.or.jp/imonline/index.html 